\title{
Kritik der Empathie - oder: Das Opfer beißt zurück: Vladimir Arsenijevićs Predator
}

Schon Nietzsche kannte die Tücken des Mitleids: „Wahrlich, ich mag sie nicht, die Barmherzigen, die selig sind in ihrem Mitleiden: $\mathrm{Zu}$ sehr gebricht es ihnen an Scham“ - so heißt es recht unmissverständlich in Also sprach Zarathustra (1968, 204-205). Nietzsches Kritik zielt auf die unverhohlene Freude derjenigen, die sich den Armen und Leidenden zuwenden - um sich dabei gut zu finden, ihre eigene Überlegenheit zu genießen. Sie profitieren - zumindest emotional, mitunter aber auch ganz praktisch - von den Opfern. ${ }^{1}$ In seiner Gefängnis-Studie Überwachen und Strafen greift Michel Foucault (1977) diesen Gedanken auf und verleiht ihm eine historische Perspektive. Das Mitleid, so Foucault, ist eine Strategie der Macht. Sie lässt Verbrecher^innen zu Opfern, zu quasi unglücklichen Straftäter^innen werden. Das Mitleid legitimiert den Strafvollzug und setzt eine profitable Maschinerie von Besserungsmaßnahmen mit Arbeitsplätzen für Jurist*innen, Aufseher*innen, Ärzt*innen und insbesondere Psycholog*innen in Gang. Ehedem tatkräftige und beängstigende Verbrecher ${ }^{\star}$ innen werden ihrer aktiven Rolle beraubt und zu Opfern von schlechten Umständen erklärt. ${ }^{2}$ Von hier aus scheint es nur noch ein kleiner Schritt bis zur Machtübernahme der Opfer selbst oder - um genauer zu sein - zum allgemeinen gesellschaftlich-politischen Siegeszug der Opferrolle, zur Verkleidung der Macht als Opfer. Diesen Wechsel zeigt uns Daniele Giglioli in seiner 2016 erschienenen Studie Die Opferfalle in prägnanter Weise. Er geht mit einer allgemeinen De-Aktivierung und Ent-Subjektivierung in Gesellschaft und Politik - mit dem Verlust von Verantwortung - einher. ${ }^{3}$

1 Ein Überblick über Nietzsches kritische Ausführungen zum Mitleid(en) findet sich in Hamburger 1985, 42-45.

2 Diese Veränderung, beispielhaft im Kapitel „Die Milde der Strafen“ beschrieben (Foucault 1977, 133-170), setzt nach Foucault im achtzehnten Jahrhundert ein und etabliert sich spätestens im neunzehnten Jahrhundert. Foucault spricht von der ,großen Transformation der Jahre 1760-1840“ (1977, 24), wobei „die großen Gesetzbücher des 18. und 19. Jahrhunderts“ das „,neue Strafsystem“ definierten (1977, 32).

3 Vgl. dazu Karsten Fischer 2006. Nach Fischer hat sich das rituelle und aktive Opfer (engl. sacrifice) im Laufe des zwanzigsten Jahrhunderts zum passiven Opfer (engl. victim) gewandelt (2006, 67-72). Während Überlebende des Ersten Weltkriegs noch argwöhnisch betrachtet und nicht selten der Kollaboration mit dem Feind verdächtigt wurden, begann sich nach dem Zweiten Weltkrieg eine neue Sichtweise zu etablieren. Viele sahen im Krieg keinen Sinn mehr und so wurden auch die Soldatenopfer obsolet. Das Erleiden von sinnloser Gewalt erschien besonders grausam, wenn Frauen, Kinder oder ältere Menschen zu Opfern wurden. Sie stellten fortan das Ideal des 
Die neuen und alten Medien zeigen sich dabei, wie sich in den genannten Studien nachlesen lässt, als geschickte Opfer-Tribünen. Ob literarische Texte oder Fotografien, Filme oder Fernsehaufnahmen - sie alle kommen als Mitleids-Katalysatoren in Betracht.

Auf eine kritische Befragung dieser Inszenierungen zielt nun gerade Vladimir Arsenijević mit seinem 2008 verfassten - als Erzählsammlung und zu Recht auch als Roman ${ }^{4} \mathrm{zu}$ klassifizierenden - Werk Predator. Arsenijević wirft einen kühlen Blick auf Empathie und Opfer-Kult. ${ }^{5}$ Der Autor mag Mitgefühl mit seinen Held^innen - mit wenigen Ausnahmen handelt es sich um Kriegsflüchtlinge anregen, jedoch nur, um diese Emotion im nächsten Moment schon wieder zu zerstören. Arsenijevićs Figuren stammen aus dem nördlichen Irak, aus Bosnien und dem Kosovo, und sie setzen sich im Verlauf des Textes gen Westen in Bewegung. Wir treffen sie in Berlin, in Barcelona, in Kopenhagen, im englischen Ilford und im US-amerikanischen Philadelphia wieder an. Zentral ist das Motiv des Durch- und Übergangs, es verdichtet sich in einem dänischen Heim für Asylbewerber*innen, durch das die meisten Protagonist*innen geschleust werden. Schon der Titel des Werks lässt vermuten, dass die Flüchtlinge unsere Zuneigung nur selten verdienen. Sowohl das englische predator (von lat. praedor - ,Beute machen', ,plündern'), das in der gleichnamigen Binnenerzählung durch den Film Predator (USA 1987, mit Arnold Schwarzenegger in der Hauptrolle) aufgerufen wird als auch das serbische predator entsprechen der (deutschen) Bedeutung eines Raubtiers. Ein aggressives Tierleben, die globalen Ausmaße des Jagens und Raubens und die Bedeutung der Medien werden durch den Titel damit aufs Kürzeste ins Spiel gebracht. Auch wenn Arsenijevićs Heldinnen zu den Leidtragenden gesellschaftlicher Krisen und politischer Machenschaften gehören - der irakisch-kurdische Konflikt und die Jugoslawienkriege der 1990er Jahre, darunter besonders der Kosovo-Krieg werden vielfach eingespielt -, so sind sie doch auf verstörende Weise aktiv. Raubtieren gleich überfallen sie Freundinnen,

passiv erleidenden Opfers dar (Fischer 2006, 71). Dass es ideale, „würdige“ im Gegensatz zu „unwürdigen“ Opfern in der von den Massenmedien geprägten europäischen Mehrheitsgesellschaft gibt, betont auch Martin Schulze Wessel (2012).

4 Die Einschätzung des Werks als Roman findet sich zum Beispiel unter https://www.laguna. rs/a979_autor_vladimir_arsenijevic_laguna.html, die Einschätzung als Erzählsammlung unter https://www.b92.net/kultura/moj_ugao.php?nav_category=559\&yyyy=2009\&mm=05\&nav_ id $=324067$.

5 Der Autor stellt mit seinem Werk nur eine Stimme innerhalb der postjugoslawischen Literatur dar. Daneben gibt es durchaus Autor^innen, die das Mitleid ihrer Leser^innen gezielt evozieren. Als Beispiel sei hier auf Slavenka Drakulićs Roman Kao da me nema (1999) [Als gäbe es mich nicht] verwiesen. 
Verwandte und Bekannte. Bevor wir uns diesen ,Opfern' und ihren aggressiven Handlungen zuwenden, sei ein kurzer Blick auf die literaturwissenschaftliche Mitleidsforschung erlaubt. Wir setzen den Akzent dabei vor allem auf kritische Studien, mit denen sich Arsenijevićs Techniken besonders gut erfassen lassen. Dazu gehört Käte Hamburgers Spätwerk Das Mitleid (1985), in dem sie den aristotelischen Ausführungen in Poetik und Rhetorik auf eigene Weise nachgeht. ${ }^{6}$

Die Tragödie, so heißt es bei Aristoteles, ist „Nachahmung einer bedeutenden Handlung [...]. Durch Mitleid und Furcht bewirkt sie eine Reinigung eben dieser Gefühle“7 (2011, 9). Durch das Mitleid mit den Schuldig-Unschuldigen, etwa Ödipus, haben die Zuschauer`innen also einen persönlichen, psychologischen Vorteil. Die Macht des Mitleids - kombiniert mit einer gewissen Passivität und ästhetischen Lust der Betrachter`innen - lässt sich an dieser Stelle bereits erkennen. ${ }^{8}$ Aber: bei Aristoteles kann nicht jede Handlung zum Ausgangspunkt einer Tragödie werden. Mitleid ist nicht immer angebracht, und Opfer müssen sich als solche qualifizieren. Bedauernswert sind nämlich nur diejenigen, die über einen tugendhaften Charakter verfügen, die gut handeln und die schuldig werden, ohne es zu ahnen. Die Empathie folgt einer vorgängigen, kognitiven Prüfung, sie ist kein unmittelbarer Impuls, kein „Elementareffekt“. ${ }^{9}$ Der ${ }^{\star}$ die Autor ${ }^{\star}$ in eines literarischen Textes wird, so könnten wir den Gedanken weiter spinnen, schon allein aus Gründen der Spannungserzeugung die Prüfung (von Schuld oder Unschuld) seiner`ihrer Heldinnen in die Wege leiten. Mitleid ist kein Gefühl, das jeder Reflexion entzogen ist, im Gegenteil: Mitleid, so konstatiert Käte Hamburger im abschließenden Teil ihrer Studie, setzt Denken und vor allem Distanz voraus. Ihr Fazit lautet: Mitleid ist „ethisch neutral“ $(1985,126)$. Diese Neutralität schließt auch das Moment der Furcht mit ein, das Hamburger als Selbstbezug der

6 Hamburger setzt sich damit im Wesentlichen von Lessings Aristoteles-Lektüre und Mitleidsbestimmung ab. Einen guten Überblick über Hamburgers Mitleids-Kritik gibt Vöhler 2007.

7 Joachim Krueger übersetzt den Passus wie folgt: „Nachahmung einer ernsthaften und in sich abgeschlossenen Handlung [...], und dadurch, daß sie Mitleid (éleos) und Furcht (phóbos) erregt, bewirkt sie die ihr eigentümliche Reinigung (he kátharsis) derartiger Affekte (pathémata)“ (1983, 219). Fuhrmann übersetzt mit „Jammer“ und „Schaudern“ (in Aristoteles 1982, 18).

8 Die Forschung ist sich nicht einig, ob hier eine Reinigung von den Gefühlen oder eine Reinigung derselben Gefühle gemeint ist.

9 Martin Vöhler weist in seiner Diskussion der „Ambivalenz des Mitleids“ besonders auf Manfred Fuhrmanns Position hin. Fuhrmann halte sich in seiner Übersetzung der aristotelischen Poetik zwar an die von Wolfgang Schadewaldt vorgeschlagenen Begriffe „Schauder“ und „Jammer“ anstelle von ,Furcht' und ,Mitleid', wende sich aber gleichzeitig gegen Schadewaldts These vom Schauder als „Elementaraffekt“ (Vöhler 2007, 41; siehe auch Schadewaldt 1956, 138). Auf die verschiedenen Übersetzungen von éleos und phóbos sowie die Aristotelische Katharsislehre geht Fuhrmann ausführlich in seiner „Dichtungstheorie der Antike“ ein (1992, 89-110.) 
mitleidenden Betrachter*innen deutet. Im Mitleid erkennen wir, dass wir in eine ähnliche Situation wie der ${ }^{\star}$ die Heldin hätten geraten können - und fürchten uns. Altruistische und egoistische Tendenzen halten sich im Mitleid, das die Tragödie evoziert, also die Waage. ${ }^{10}$

In eine ähnliche Richtung argumentiert der Literatur- und Kognitionswissenschaftler Fritz Breithaupt. In seinem 2015 erschienenen Artikel „Empathic Sadism: How Readers Get Implicated“ geht er dezidiert auf die Empathieentwicklung im Leseprozess ein. Breithaupt stellt die These auf, dass Empathie keine physischen oder psychischen Schmerzen beinhalte, folglich auch kein Mitleiden sei, sondern durchaus als angenehm empfunden werden könne. Er nennt dieses Phänomen, das er im Übrigen nicht nur auf die fiktive Welt beschränkt, empathischen Sadismus (2015, 440-442; 2017, 149-186). Fiktive Welten sind besonders geeignet, empathisch-sadistische Reaktionen unter der Leserschaft hervorzurufen.

In Die dunklen Seiten der Empathie entwickelt Breithaupt seine These weiter und entwirft ein plausibles Modell, um die Funktionsweisen der Empathie zu erklären (2017, 79-124). Er geht davon aus, dass Empathie nicht nur der Logik eines Reiz-Reaktions-Modells folgt, sondern komplexeren Mechanismen gehorcht. Breithaupt identifiziert Blockaden, Durchbrechungen und Kanalisierungen, um die Regulierung des Empathie-Flusses zwischen der beobachteten Figur, dem sogenannten „Empathie-Erreger“, und dem Leser ${ }^{11}$, dem sogenannten „hyper-empathischen Beobachter“ zu beschreiben. Kontrollmechanismen sind nötig, um die Gefahr eines Empathie-Exzesses und - damit verbunden - eines totalen Selbstverlusts zu vermeiden (2017, 80). Diese „Empathie-Blockaden“ (2017, 85-100) sind stets (also auch in der Wirklichkeit) vorhanden, doch können sie unter bestimmten Bedingungen und durch spezielle „Empathie-Auslöser“ (2017, 93-94) durchbrochen werden. Die literarische Verfasstheit eines Textes, seine Künstlichkeit begünstigt solche Bruchstellen, gleichzeitig wissen wir - auch wenn wir uns im Leseprozess mitunter selbst vergessen ${ }^{12}$ - um diese Bedingung. Breithaupt geht in seiner Argumentation zumindest implizit mit Hamburger einher: Die Distanz zwischen den Leser*innen und den leidenden Figuren beruht auf der Tatsache, dass die Ersteren in keinem ,echten“ Verhältnis zu den Letzteren stehen. Breithaupt bringt diese Voraussetzung - unter Berufung auf Suzanne

10 So lautet das Fazit von Vöhlers Hamburger-Lektüre (2007, 42).

11 Da der Begriff des Lesers eher abstrakt zu verstehen ist und auch der besseren Lesbarkeit halber wird hier und im Folgenden auf die feminine Singular-Form verzichtet. Konkret sind immer beide Geschlechter gemeint.

12 Das gilt besonders dann, wenn wir dem Realitätseffekt (Barthes 1976, 84) aufsitzen, und trifft weniger für literarische Werke zu, die deutliche Illusionsbrechungen (wie Predator) enthalten. 
Keen ${ }^{13}$ - sehr treffend auf den Punkt: „Readers participate vicariously in characters and their situations, without having to fear that the fictional characters will turn around and ask them for money“ $(2015,442)$.

Literatur begünstigt Empathie auch durch ihre zeitliche Rahmung: Die Erzählung verspricht zu einem Ende zu kommen. Dieses Versprechen motiviert den Leser, sich ganz in eine oder mehrere Figuren imaginativ und emotional hineinzufühlen (Breithaupt 2017, 96). ${ }^{14}$ Um Empathie aufrecht $\mathrm{zu}$ erhalten sind also nach Breithaupt Bewegung, Veränderung und eine zeitlich absehbare Auflösung nötig (2015, 442). Die Auflösung kann negativ sein und den Tod des Helden oder der Heldin bedeuten, wie dies in der Tragödie der Fall ist, oder aber positiv. Eine positive Lösung sieht Breithaupt in Lisa Zunshines Figurentypus des „sadistic benefactor“ (2012, 45-53), der Gefallen daran findet, andere schwer leiden zu sehen, um ihnen dann zu großem Glück und zu einer deutlichen Verbesserung ihrer Lage zu verhelfen (Breithaupt 2015, 444-445). Diesen Mechanismus erkennt Breithaupt auch im Verhältnis zwischen Figur und Leser wieder, insbesondere bei Versuchungsgeschichten oder beim Bildungsroman (2015, 444-445).

Erzählungen, die sich der Opferperspektive nähern oder über Opfer berichten, können unter der Leserschaft eine besondere Form der Empathie, eine „advocative exploitative empathy“ anregen (Breithaupt 2015, 445). In diesem Falle wird der Leser zum Verteidiger des leidenden Opfers und, wenn dem Opfer Unrecht angetan wurde, zum Ankläger gegen Ungerechtigkeit und Grausamkeit. Nicht zu vergessen ist jedoch, dass der Leser Gefallen an dieser Rolle finden kann. Wie Breithaupt richtig bemerkt, wird hier ein unauflösbares Paradoxon erkennbar, denn so sehr sich der empathische Leser eine Verbesserung der Lage für das leidende Opfer wünschen mag, so sehr ist er in seiner Rolle als Verteidiger des Opfers auf dessen leidvollen Zustand angewiesen $(2015,445) .{ }^{15}$ Das Leid ist

13 Suzanne Keen argumentiert in ihrer Monografie Empathy and the Novel (2007, 28-35), dass man beim Lesen von fiktiver Literatur eher Empathie empfindet als in einer Situation, in der man direkt angesprochen und um Hilfe gebeten wird.

14 Breithaupt führt in diesem Zusammenhang einen interessanten Vergleich mit einem Alltagsphänomen an. Wissenschaftliche Untersuchungen legen die Annahme nahe, dass manche Menschen gegenüber akut erkrankten Patienten eher Empathie zeigen als gegenüber chronisch erkrankten (2017, 96). Das Mitleid scheint eine begrenzte Ressource zu sein, die sich bei übermäBiger Beanspruchung in Gleichgültigkeit oder sogar in Ressentiment verkehren kann.

15 Eine Beobachtung, die von Foucault (1977) in seiner Analyse des Gefängnissystems geteilt wird: Um Mitleid (mit den Verbrecherinnen) aktiv werden zu lassen, müssen sich die Gefängnisse stetig füllen, müssen Verbrechen vor allem kontinuierlich begangen - und nicht umgekehrt: verhindert oder die Verbrecher`innen gar geheilt werden. Dergestalt reproduziert sich das Gefängnissystem selbst. 
die Bedingung von Empathie und Gerechtigkeitsempfinden. Die „Ambivalenz“ (Hamburger) und die „Schamlosigkeit“ (Nietzsche) des Mitleids klingen in dieser Bestimmung erneut mit an.

Es gehört zu den besonderen Leistungen von Vladimir Arsenijević, dass er den quasi ökonomischen Mechanismus des Mitleidens auf gekonnte Weise inszeniert und demaskiert. Der Autor von Predator spielt mit den Opfer-Rollen, und er rechnet gleichzeitig mit den empathischen Profiteur`innen, seinen Leser^innen ab. $\mathrm{Zu}$ den Strategien Arsenijevićs zählt zunächst einmal - ganz im Sinne der russischen Formalisten - die „Bloßlegung des [literarischen] Verfahrens“. ${ }^{16}$ Der Text ist zuvorderst ein Text, kein Abbild der Wirklichkeit und wird uns auch als ein solcher, d.h. als eine künstliche Welt, vor Augen geführt.

Schon die Komposition von Predator zielt auf diesen Effekt, denn die erste Erzählung verspricht die letzte zu sein - „Poslednja epizoda Oahu Džima \# 1“ (Arsenijević 2009, 7-38) [Die letzte Episode von Oahu Jim \# 1] ${ }^{17}$-, während die letzte Erzählung dieses scheinbar zirkuläre Unternehmen konterkariert, indem sie eine Fortsetzung bietet: „Poslednja epizoda Oahu Džima \# 2“ (2009, 221-242) [Die letzte Episode von Oahu Jim \# 2]. Im Innern des Buchs geht es weder zirkulär noch logisch-chronologisch voran. Wir treffen auf zahlreiche Szenen-, Genre-, Perspektiv- und Stilwechsel. Lässig-provokativ, im Jargon formulierte Pseudo-EMails eines serbischen Migranten wechseln sich mit klassischen Erzählpartien $\mathrm{ab}$, in denen wir mit der Innensicht von Figuren, zum Beispiel mit ihren Ängsten, aber auch mit der Leseranrede des allwissenden Erzählers konfrontiert werden. Multiperspektivisch gestaltete Szenen stehen einmaligen Ereignissen gegenüber. Zahlreiche Figuren treten in mehreren Erzählungen und in verschiedenen Kontexten auf - dazu gehört der jesidische Kurde Nihil Baksi -, manche dagegen erscheinen nur einmal und verschwinden, wie der Kosovo-Albaner Dren Kastrati, ohne eine Spur zu hinterlassen. Einige Nebenfiguren - Vanja, ein Mitglied der Rockband GSG9, und die Schauspielerin Marija Pavlović mit ihren Kindern Vera und Jovan - haben sogar ein erstaunlich langes ,Leben': Sie entstammen Arsenijevićs Werken der 1990er Jahre (dem preisgekrönten Debütroman U potpalublju und seiner Fortsetzung Ti i ja, Anđela ${ }^{18}$ ), womit ihre Künstlichkeit eigens

16 Siehe den Begriff zum Beispiel bei Viktor Šklovskij 1929, 178, 180, 241.

17 Sofern nicht anders vermerkt, stammen die Übersetzungen von den Verfasserinnen - F. M. und A. Z.

18 Arsenijević hatte in den 1990er Jahren eine Tetralogie mit dem Titel Cloaca maxima geplant, die sich über lange Zeit in den ersten beiden Bänden (U potpalublju [1994] und Ti i ja, Anđela [1997]) erschöpft hatte, 2018 nun aber mit einem dritten Teil (Ka granici) fortgesetzt wurde. Mit Abstrichen könnte man auch einige Werke der 2000er Jahre wie etwa Meksiko: Ratni dnevnik (2000) als partielle Realisierungen des ursprünglichen Projekts interpretieren. 
zur Schau gestellt wird. Die Figuren verdanken ihre Existenz ganz offensichtlich den Fantasien des Autors. Diesen herkömmlichen Trick, mit dem auch schon Cervantes arbeitet, ergänzt Arsenijević durch postmoderne Techniken: Fiktion wird mitunter von Fakten durchsetzt, und das Spiel erhält eine ernste Note: Das Asylzentrum im dänischen Avnstrup existiert jedenfalls noch heute. ${ }^{19}$

Predator, so können wir fürs Erste festhalten, stellt eine komplexe literarische Konstruktion dar, die sich in den Vordergrund schiebt und dergestalt das Denken der Leser ${ }^{\star}$ innen in Gang setzt. Es gilt die Handlungsfäden zu erkennen, Figuren und Schauplätze zu identifizieren, eine Fülle an Informationen zu verarbeiten - ohne dass eine allzu große Identifikation mit dem Erzählten erlaubt wäre. Wie die Heldinnen, so sind auch die Leser`innen ständig unterwegs, mit jeder Erzählung müssen sie sich auf neue Kontexte und Abenteuer einstellen. Der Autor schafft es aber auch, seine Leserschaft emotional in den Bann zu ziehen. Und hier kommt das Mitleid mit all seinen paradoxen Eigenschaften ins Spiel. Unser Sadismus wird uns gewissermaßen vor Augen geführt, wir werden ertappt, wenn wir genüsslich dem Leiden der anderen zusehen und enttäuscht, wenn wir auf eine Fortsetzung dieses bequemen und darüber hinaus spannend aufgebauten Gefühls gehofft haben. Es ist vor allem der emotionale Wechsel, der sowohl fasziniert als auch verstört. Die Geschichten von Nihil Baksi ${ }^{20}$ und Dren Kastrati ${ }^{21}$ seien als Beispiel für Arsenijevićs Empathiekritik unter die Lupe genommen.

Predator setzt mit dem ersten persönlichen Treffen zwischen James Rice alias Oahu Jim, einem bekannten amerikanischen TV-Abenteurer, und Nihil Musa Baksi, einem irakisch-kurdischen Flüchtling und Kannibalen, ein. Die beiden haben sich im Internet in einem Forum für Vorarephilie und Anthropophagie kennen gelernt und möchten nun ihre Interessen in die Tat umsetzen: Im Falle von Jim handelt es sich um sexuelle Fantasien; Nihil Musa will seine aus der Not geborene Sucht nach Menschenfleisch stillen. Zum kannibalischen Akt, d.h. zum Verspeisen des Fernsehstars durch den Flüchtling, kommt es allerdings erst am Ende des Buches, wenn die Oahu-Jim-Geschichte wieder aufgenommen und die klassische Opfer- und Täter-Dichotomie mit Ironie und bissigem Humor konterkariert wird. Auf das Mitgefühl der Leserinnen legt es der Autor in dieser Rahmenerzählung gar nicht an, die Opfer- und Empathiekritik, die sich durch den ganzen

19 Siehe https://www.rodekors.dk/det-goer-vi/roede-kors-asyl/asylcentre/center-avnstrup.

20 Ausgeführt vor allem in den Erzählungen „Poslednja epizoda Oahu Džima \# 1“, „Zemljaci“, „Poslednja epizoda Oahu Džima \# 2“ (Arsenijević 2009, 5-38, 83-146, 147-194, 221-242) [Die letzte Episode von Oahu Jim \# 1, Landsleute, Die letzte Episode von Oahu Jim \# 2].

21 Ausgeführt vor allem in der Erzählung „Neukorenjenost“ (Arsenijević 2009, 39-74) [Wurzellosigkeit]. 
Roman zieht, erreicht in „Oahu Jims letzter Episode \# 2“ sogar einen Höhepunkt. James Rice, das Opfer, das sexuelle Befriedigung in seiner Opferrolle findet, folgt Nihil, dem Predator, in den Keller, legt sich dort auf den Boden und bringt einen Dialog in Gang, der an ein unbeholfenes, sexuelles Herantasten von Teenagern erinnert:

„Da li je tako bilo i ranije?“ oglasi se napokon Žrtva nešto mirnijim glasom. „Ranije?“ tiho na to uzvrati Nihil Musa Baksi ne podižući pogled.

„Ranije“, kaže Žrtva. „S drugim ... S drugima.“

„Ne volim da pričam o tome“, kaže Nihil Musa suvo.

Ipak, nekoliko trenutaka kasnije tiho doda: „Nije. Svaki put je drugačije.“

(Arsenijević 2009, 232)

[,War es auch früher so?“, fragt das Opfer etwas ruhiger.

„Früher?“, entgegnet Nihil Musa Baksi leise ohne aufzublicken.

„Früher“, sagt das Opfer. „Mit dem anderen ... Den anderen.“

„Ich mag nicht darüber sprechen“, sagt Nihil Musa trocken.

Kurze Zeit später fügt er aber dennoch leise hinzu: „Nein. Jedes Mal ist es anders.“]

Nachdem sich Nihil mehrere Male versichert hat, dass das „Opfer“ wirklich „bereit“ sei, zu sterben, äußert Jim, der das zukünftige „Fleisch“ verkörpert (sein Kennwort im Internetforum ist „meat“), den Wunsch zu verbluten. Er möchte sich beim Sterben selbst zusehen. Diesen melodramatischen Todeswunsch erfüllt ihm Nihil aber nicht, er greift zur Pistole und erschießt sein Gegenüber schnell und unspektakulär:

Žrtva je, kako se to od nje i očekivalo, samo nemoćno zakolutila očima i otplovila zauvek. [...] Eto, koliko je sve to u stvari lako i jednostavno. Ah ah ah, jedan dva tri i - gotovo! Slatka smrt je stigla. Konačno. Nema više Džejmsa Rajsa, nema u celom svetu voljenog i gledanog Oahu Džima, malog Džima iz Filadelfije, grada bratske ljubavi i najmasnije hrane na celom jebenom svetu. Hteo je da bude vinovnik sopstvenog umiranja, punopravni učesnik $\mathrm{u}$ njemu do poslednjeg časa, a, eto, nasamaren je, događa se i to, nasamaren je i umlaćen kao najobičnije goveče, lako, brzo i efikasno, uz izrazito racionalan, minimalan utrošak energije od strane iskusnog Dželata koji je svoj posao obavio toliko mirno i staloženo da se nije čak ni oznojio. (Arsenijević 2009, 234)

[Das Opfer verdrehte nur schwach die Augen und trieb für immer davon, wie man das von ihm erwartete. [...] Schau da, wie leicht und einfach das doch eigentlich alles ist. Ha, ha, ha, eins, zwei, drei und - fertig! Der süße Tod ist da. Endlich. Es gibt keinen James Rice mehr, es gibt den geliebten Oahu Jim, dessen Sendungen auf der ganzen Welt geschaut werden, nicht mehr, den kleinen Jimmy aus Philadelphia, der Stadt der Bruderliebe und des fettigsten Essens auf der ganzen verdammten Welt. Er wollte der Initiator seines Sterbens sein und der vollberechtigte Teilhaber bis zum letzten Augenblick, doch schau da, er wurde hereingelegt, auch das passiert, er wurde hereingelegt und wie ein ganz gewöhnliches Rind abgetan, leicht, schnell und effizient und das bei deutlich rationellem und mini- 
malem Energieaufwand seitens des erfahrenen Scharfrichters, der seine Arbeit so ruhig und besonnen verrichtete, dass er nicht einmal ins Schwitzen kam.]

Die Täter-Opfer Dichotomie wird hier auf heiter-lakonische Weise ebenso auf die Schippe genommen wie die Lust am Opfer, die sadistische Empathie, die Lust des Opfers an sich selbst und das Pathos des Sterbens. Nicht nur wird James am Ende getäuscht, auch der Text hält nicht, was er den Leser`innen zu Beginn verspricht: einen bittersüßen Tod mit Empathiepotenzial. Stattdessen räumt Nihil Baksi, nachdem er sich lange von seinem Opfer ernährt und seine Sucht überwunden hat, alle Spuren aus dem Weg und setzt sich gleichsam heiter nach Kalifornien ab.

Nihil Baksi wird uns aber nicht nur als kühler Kannibale vorgeführt, sondern auch als bedauernswerter Junge. Sorgfältig baut Arsenijević diese Empathiefährte in der zentralen, titelgebenden Erzählung seines Werks auf (2009, 83-146). Bis zu diesem Zeitpunkt - falls wir den Text in der vom Autor angebotenen Reihenfolge lesen - sind wir mit Nihil erst vorsichtig bekannt gemacht worden. Der kurdische Held mag uns ein wenig suspekt sein, immerhin sucht er im Internet nach Menschenfleisch, aber wir treten ihm - der nunmehr als Kind und Jugendlicher präsentiert wird - doch mehr oder weniger unbedarft entgegen. Nihil wird aller Wahrscheinlichkeit nach unser Herz erweichen: Er muss erleben, wie sein Vater vom irakischen Geheimdienst vor seinen eigenen Augen erschlagen wird, er wird selbst verschleppt und brutal gefoltert, überlebt knapp und flieht mit seiner Familie ins kurdisch besetzte Halabdscha. Dort sterben seine Mutter und die beiden Schwestern bei einem Giftgasangriff der irakischen Luftwaffe. Nihil kann sich mit seinem kleineren, von seiner Familie adoptierten Bruder Musa gerade noch ins nahegelegene Gebirge retten.

Bis zu diesem Zeitpunkt erfüllt Nihil Baksi alle Anforderungen, um ein „würdiges“ Opfer zu sein und Empathie zu ermöglichen. Er ist jung und unschuldig, fast noch ein Kind, wurde aufs Schwerste gefoltert und hat allein aufgrund seiner ethnisch-religiösen Zugehörigkeit fast alle Familienangehörigen verloren. Er steht förmlich vor dem Nichts, eine Situation, die sein Name vorherzusagen scheint. Nihil weist außerdem positive Charakterzüge auf, er ist mutig und kümmert sich um seinen kleinen, naiven Adoptivbruder. Die schlechte politische Situation tut ein Übriges. Es ist unschwer zu erkennen, welches die ,böse‘ und welches die ,gute‘ Seite ist. Auf der einen stehen ein despotischer Diktator und seine brutalen Repräsentant`innen, auf der anderen die vom Staat unterdrückten und terrorisierten Minderheiten der Kurd`innen und Jesid`innen.

Doch an einem bestimmten Punkt der Erzählung löst sich das Mitleid höchstwahrscheinlich auf, unsere Emotionen finden keine positiven Anhaltspunkte mehr. Was bleibt, ist eine Figur, die sich so schuldig gemacht hat, dass sie als 
Empathieobjekt nicht mehr funktioniert. Das geschieht in dem Augenblick, in dem das Opfer zum Täter wird.

In dieser Szene herrschen Extrembedingungen. Nihil und sein Ziehbruder Musa sind physisch erschöpft und befinden sich in den Bergen, wo es kalt ist, ihnen niemand hilft und sie der Hunger plagt. Beide schlafen aus Erschöpfung ein. Nihil wacht als Erster wieder auf. Da überfällt ihn plötzlich ein Gedanke. Er setzt die Idee, die ihn nicht mehr loslässt, in die Tat um und erschlägt seinen kleinen Bruder.

Diese Handlung und ihre Brutalität lassen sich auch unter Berücksichtigung der lebensbedrohlichen Situation des Helden und seiner Erinnerung an Folter und Hunger nicht rechtfertigen. Dafür sorgt der Erzähler, indem er ein klares Urteil spricht:

A Nihil Baksi, Predator, Strašni Bog koji presuđuje bez milosti i hrani se telima onih koji u njega najviše veruju, još jednom podiže kamen s kog kaplje krv i gusta bela sluz, pa ga ponovo zariva $u$ isto ono mesto $u$ koje je maločas udario. [...] Tu spusti lešinu do sebe, sedne prekrštenih nogu, nadvije se nad nju i zarije zube u meki prevoj vrata. [...] Kad pomiri prvu glad, Nihil - jer sad je to zaista ponovo Nihil, iako zauvek i neporavljivo izmenjen - Nihil, dakle, opet spava, sanja svoje mukle, uvek iste snove o lutanju bez kraja i konca, pa se budi u tami i smeši u sebi, zadovoljan. (Arsenijević 2009, 128-129)

[Nihil Baksi aber, der Predator und grausame Gott, der unbarmherzig urteilt und sich von den Körpern derjenigen ernährt, die am meisten an ihn glauben, hebt den Stein, von dem Blut und dickflüssiger weißer Schleim tropft, noch einmal und bohrt ihn erneut in die gleiche Stelle, auf die er kurz davor schon einmal geschlagen hat. [...] Hier lässt er den Kadaver neben sich fallen, setzt sich im Schneidersitz hin, beugt sich über ihn und gräbt seine Zähne ins weiche Joch des Halses. [...] Nachdem Nihil - denn das ist jetzt wirklich wieder Nihil, obwohl für immer und unwiderruflich verändert - den ersten Hunger gestillt hat, schläft er also wieder ein, träumt seine dumpfen, immer gleichen Träume vom endlosen Herumirren, wacht in der Dunkelheit auf und lacht in sich zufrieden.]

Die Figur hat sich völlig verändert und sich vom potenziellen Mitleid der Leser`innen befreit, doch sie bleibt für eine Weile die einzige IdentifikationsGestalt in der Erzählung. Uns wird nichts anderes geboten, als Nihil, dem Mörder und Kannibalen, der nichts mehr mit dem einstigen Opfer, dem unschuldigen, gefolterten 16-jährigen Jungen, gemein hat, auf seinem weiteren Weg zu folgen.

Die Strategie der Zerstörung von möglichen Mitleidfiguren, die Arsenijević in mehreren Variationen durchspielt, hat zum Ziel, auch auf struktureller Ebene Empathiekritik zu üben. Falls die Leser^innen Mitleidsgefühle entwickeln - und der Autor regt sie dazu an -, so werden sie immer wieder ent- und getäuscht, bis sie - vielleicht - nicht mehr in die Opferfalle tappen. Allzu erfahren und kognitiv gerüstet sind die Leser^innen, wenn sie sich an die zweite Erzählung des Zyklus, „Neukorenjenost“ (Arsenijević 2009, 39-74) [Wurzellosigkeit], wagen, 
aber noch nicht. Die Opferfalle wird aller Wahrscheinlichkeit nach zuschnappen, denn der Held Dren Kastrati, ${ }^{22}$ der dem militärisch-politischen Konflikt in seiner kosovarischen Heimat entflohen ist, kommt tragisch zu Tode. Er hat bis zu seinem Verschwinden aus dem Text keinerlei Schuld auf sich geladen, stellt also ein wahrhaft „würdiges Opfer“ dar, und sogar das böse Gegenüber - ein Serbe - lässt sich für die Rezipient*innen ohne Probleme ausmachen. Relativierend wirkt allerdings, dass die Fabel um Kastrati in ein opulentes mediales Spiel, ja man könnte sagen in eine offene Demonstration und Kritik des medial inszenierten Mitleidmarktes eingebettet ist. Obwohl alle Erzählungen medial verwurzelt und miteinander verflochten sind, obwohl alle Held^innen auf ihrem Leidensweg von Film- und Fernsehbildern, von Fotos und kulturellen Events begleitet oder durch selbige zum Leiden angeregt werden, ${ }^{23}$ ist die Dekonstruktion der Medien in „Neukorenjenost“ besonders auffallend. Gleichsam im Vorübergehen und mit einer heiteren Note versehen, deckt Arsenijević die Strategien von Schriftstellern - darunter nicht zuletzt seine eigenen -, von NGO-Mitgliedern, linken Aktivisten, Fernsehjournalisten, Fotografen und Organisatoren europäischer Dokumentations-Wettbewerbe auf. Sie alle brauchen das Opfer, mit Opfern macht man Geschäfte, man legitimiert den eigenen Job, die Fernsehsendung, den hochdotierten Preis, man steigert das Prestige der Zeitschrift und die Verkaufschancen für das eigene Manuskript. Dieses Spiel mit den Medien und deshalb auch mit der schriftstellerischen Fiktion zeigt sich bereits darin, dass „Neukorenjenost“ mit einer Figur aufwartet, der serbischen Schriftstellerin Marija Pavlović, die ihrerseits einen Roman mit dem Titel Neukorenjenost geschrieben hat, ${ }^{24}$ und auch die dokumentarische Foto-Serie, die das Ende von Arsenijevićs Binnengeschichte ausmacht, trägt den - wie es heißt - „ausgesprochen enigmatischen“ ${ }^{25}$

22 Der im Deutschen leicht komisch und verunglimpfend wirkende Name Kastrati ist die albanische Version von Kastrioti, dem Namen eines alten Adelsgeschlechts, dem auch der berühmte Skanderbeg zugehörte („Kastrioti“). Der Name Kastrati ist in der heutigen albanischen Öffentlichkeit durch die zahlreichen Tankstellen der Firma Kastrati allgegenwärtig.

23 So kommt etwa Oahu Jim zu seinen sexuell inspirierten Opferphantasien, als er im zarten Alter von zehn Jahren in der Zeitschrift Home \& Away einen dicken Forscher abgebildet sieht, der von Afrikanern in einem Topf auf offenem Feuer (zusammen mit Suppengrün) gekocht wird und dabei glücklich lächelt (Arsenijević 2009, 16). Die Videokassette mit dem Film Predator begleitet den jugendlichen Nihil Baksi auf seiner Flucht. Sie ist das einzige Gut, das er aus seiner Heimat retten konnte.

24 Allerdings war die Heldin erfolgreicher als der Autor, sie hat ihren Roman Wurzellosigkeit nämlich schon ins Deutsche übersetzen lassen und beim Fischer Verlag untergebracht, während der Autor nur die deutsche Übersetzung der Erzählung „Wurzellosigkeit“ in Swartz’ Anthologie (2007, 25-46) publizieren, nicht aber seinen Roman Predator auf Deutsch herausbringen konnte. 25 Sofern nicht anders vermerkt, stammen die Übersetzungen von den Verfasserinnen - F. M. 
Titel „Wurzellosigkeit“ (Arsenijević 2009, 74). ${ }^{26}$ Die Wurzel- oder Heimatlosen so scheint uns der Text zu sagen - lassen sich vielfach vermarkten, und auf provokativ-ironische Weise beteiligt sich auch der Autor an diesem Spiel. ${ }^{27}$ Gerade durch seine Einmischung verhindert er, dass wir uns mit dem Erzählten identifizieren. Er fordert vielmehr die Aktivität seiner Leser^innen, ihr Kritikvermögen heraus, und Arsenijević zieht dabei alle Register. Gleichsam nebenbei setzt er ein klassisches, seit langem aber aus der Mode gekommenes Verfahren ein: sein Erzähler tritt mitunter in einen direkten Dialog mit seinem impliziten Gegenüber: „da samo jednom udahneš vazduh, čitaoče, i evo, nisi uspeo ni da ga ispustiš iz pluća, a oni su već tu, u Berlinu“ $(2009$, 56) [du musst nur einmal die Luft einatmen, Leser, und, siehe da, du hast sie noch nicht einmal aus deinen Lungen gelassen, da sind sie schon hier, in Berlin].

Daneben organisiert der Autor die Handlung in Form einer filmisch anmutenden Parallelmontage - um nur zwei Beispiele seiner Intervention zu nennen: Dren Kastrati, als Kriegsflüchtling mit einem Ausweis der Genfer Konvention ausgestattet, macht sich unter dem Einfluss seiner Freundin Lola auf den Weg nach Berlin, und auch Marija Pavlović kommt - parallel geschaltet - gerade in Berlin an, um an einer NGO-Veranstaltung teilzunehmen. Die beiden Handlungsfäden verbinden sich schließlich in einer grandiosen Tumult-Szene, die einem durch und durch inszenierten Krieg entspringt: den alljährlichen, quasi ritualisierten Mai-Krawallen der linken autonomen Szene in Berlin. Die literarischen, filmischen und fotografischen Verfahren, aber auch die Regeln von Festen oder Wohltätigkeitsveranstaltungen, die sich alle um das Opferdasein drehen, werden also in vielfältiger Weise als Fiktion entlarvt und als verlässliche Zeichen für die Wirklichkeit in Frage gestellt.

Dabei sind die ökonomischen Manöver des Berliner Zentrums für interkulturellen Dialog ein besonderes Objekt des Autor-Spotts. Das Zentrum lädt Marija

und A. Z.; die deutsche Übersetzung von Jelena Petrović (im Inhaltsverzeichnis fälschlicherweise als Telend Petrović angegeben), die sich in Swartz' Anthologie findet, wurde vermutlich in großer Eile angefertigt, sie enthält viele Auslassungen und wird aus diesem Grund für den vorliegenden Aufsatz nicht verwendet (Arsenijević 2007, 25-46).

26 Aus der Erzählung „Zemljaci“ (Arsenijević 2009, 147-194) [Landsleute] erfährt man, dass die Dänin Hannah Nielsen für die Serie verantwortlich zeichnet, sie hat darüber hinaus Dokumentarfilme über das Asylzentrum Avnstrup und über die Belgrader Studentenproteste der 1990er Jahre gemacht (Arsenijević 2009, 154, 167).

27 Er macht sich nicht nur als Erfinder der Erzählung „Wurzellosigkeit“ bemerkbar, sondern er hat noch weitere Literatur anzubieten: seine Heldin Marija Pavlović hat er aus einem Vorgängerroman von Predator (Ti i ja, Anđela [1997]) entnommen und sie also eigenmächtig zu neuem Leben erweckt. 
Pavlović - in Berlin gerade aus Budapest und nicht aus dem bedrohten Belgrad eingetroffen - zusammen mit dem kosovarischen Schriftsteller Fatmir Berišaj, seinerseits aus Graz angereist, wo er schon lange als Stadtschreiber tätig war und noch immer ist, zu einem öffentlichen Diskussionsabend ein. Die Veranstaltung findet unter dem Titel „Serbia vs. Kosovo: No Acceptance/No Repentance“ statt. Als Repräsentantin des Zentrums tritt Ulrike Krüger auf. Ulrike ist ein echter ,Gutmensch' und deshalb mit einem gesunden Nützlichkeitsdenken ausgestattet. Auf der Toilette in Marijas Wohnung sitzend reflektiert sie über den mäßigen Output ihrer Opfer-Suche:

dobili [su] dvoje elokventnih i iskusnih igrača, ali ujedno i nedovoljno krvi, znoja i suza, ali krv, znoj i suze jesu upravo ono što Centru treba [...], Marijina melanholija i Fatmirova ironija nisu dovoljna i odgovarajuća nadoknada za to, za onu pravu stvar, za grozomorno iskustvo s lica mesta, ali šta se tu može, vremena su takva, utakmica je jaka, puno je igrača u kaznenom prostoru nevladinog sektora, gužva ispred gola neopisiva, ne postižu se uvek spektakularni rezultati, i Ulrike to sedi na wc-šolji u Marijinom toaletu, veoma dobro zna. (Arsenijević 2009, 54 [Hervorhebung im Original])

[sie haben zwei eloquente und erfahrene Spieler erhalten, aber deshalb auch zu wenig Blut, Schweiß und Tränen, wo doch Blut, Schweiß und Tränen genau das sind, was das Zentrum braucht [...], Marijas Melancholie und Fatmirs Ironie sind keine ausreichende und angemessene Entschädigung für die wahre Sache, die Erfahrung des Grauens von Angesicht zu Angesicht, aber so sind eben die Zeiten, der Wettkampf ist hart, viele Spieler halten sich im Strafraum des NGO-Sektors auf, das Gedränge vor dem Tor ist unbeschreiblich, spektakuläre Resultate sind selten und Ulrike weiß das, während sie bei Marija auf der Kloschüssel sitzt, ganz genau. ${ }^{28}$

Aber: Bei diesem Spiel bleibt Arsenijević nicht stehen. Brisant ist die Erzählung zunächst einmal, weil der Autor - darin stellt er in der serbischen Literaturszene eine Ausnahme dar - eigens auf den Kosovo-Krieg zu sprechen kommt und damit auch implizit die nationale Opfer-Erzählung der Serb^innen untergräbt. ${ }^{29}$ Darüber hinaus stellt er - ohne sich auf eine Seite zu schlagen - die Opferkon-

28 Mit Blick auf einen echten Krieg und echte Opfer sind der Begriff „Spieler“, der Vergleich mit dem Fußballspiel und die Situation auf der Toilette durchaus provokativ; dabei werden die notwendigen Opfer-Ingredienzen klar benannt und in ihrer Instrumentalisierung auch kritisiert. 29 Diese Opfererzählung besagt, dass der Kosovo „heiliges, serbisches Land“ sei, beruhend auf der Kosovo-Schlacht von 1389 (in der das serbische Heer dem osmanischen unterlag), sich folglich der serbische Fürst Lazar mit seinen Truppen stellvertretend für das abendländische Christentum (und ähnlich wie Christus für die Menschheit), geopfert ‘ habe. Diese Position vertritt in „Neukorenjenost“ eine deutlich negativ markierte Figur: „Kosovo [...] je sveta srpska zemlja“ (Arsenijević 2009, 66) [Der Kosovo ist heiliges serbisches Land]. 
kurrenz von Serb^innen und Albaner`innen samt den vorgefassten Meinungen der westeuropäischen $\mathrm{NGOs}^{30}$ zur Schau. Brisant ist die Erzählung schließlich vor allem, weil - in einem sehr kurzen Telefongespräch - die (mögliche, ja sogar wahrscheinliche) Brutalität des realen Geschehens aufblitzt. Für einen Augenblick wird die Illusion nicht durchbrochen; die Leser`innen werden vielmehr in die Wirklichkeits-Illusion eingelassen und mit der Vermutung konfrontiert, dass die Telefonleitung die Wahrheit vermitteln könnte, den Tod, oder, um genauer zu sein, die Ermordung eines Menschen: Nach seiner Ankunft in Berlin versucht Dren Kastrati mehrfach erfolglos, seinen Vater im Kosovo per Telefon zu erreichen. Nach mehreren Wochen Unterbrechung scheint die Telefonverbindung wieder zu funktionieren:

Tek da bi se smirio, da bi odstranio oblak tamnih slutnji koji se pred sumrak nadvio nad njim, [...] iz govornice na Šenhauzerale, Dren zove kući, u Prizren, i dah mu zastaje kak protivno očekivanijima dobije vezu [...], a kad začuje zvuk podizanja slušalice, kratko statično krčanje i napokon nesigurni muški glas koji na čistom srpskom kaže: Da? - on, u panici i očajanju, naglo spušta slušalicu. (Arsenijević 2009, 59 [Hervorhebung im Original])

[Nur um sich zu beruhigen, um die Wolke düsterer Vorahnungen, die sich in der Dämmerung vor ihm aufgebaut hat, zu vertreiben, ruft Dren [...] aus einer Telefonzelle in der Schönhauser Allee zu Hause, in Prizren, an und sein Atem stockt, als er unerwartet eine Verbindung bekommt [...] und als er hört, wie auf der anderen Seite der Hörer abgehoben wird - ein kurzes statisches Rauschen und endlich eine männliche Stimme die auf Serbisch Ja? sagt - legt er in Panik und Verzweiflung auf.]

Die Pause, das panische Auflegen des Hörers und die Unterbrechung des Gesprächs signalisieren das Schweigen der (echten) Opfer, denn für Dren - und letztlich auch für die politisch-historisch informierten Leser*innen - ist der Hintergrund der Frage „Da?“ [Ja?], also das nicht erzählte Geschehen, klar: das väterliche Haus im Kosovo wurde von Serb^innen erobert, der Vater ist nicht mehr dort, ja er ist vielleicht überhaupt nicht mehr unter den Lebenden, und diese Ahnung wird durch einen zweiten Anrufversuch bestätigt: „Da ti pravo kažem“, gibt der Serbe nun zu verstehen, „što se njega [tvog oca] tiče, tu nemaš više šta da brineš“ (Arsenijević 2009, 65-66) [Um ehrlich zu sein, was ihn [deinen Vater] betrifft, brauchst du dir keine Sorgen mehr zu machen].

30 Auch die westeuropäische öffentliche Meinung wird hier kritisiert, so unterstellen die westeuropäischen Linken (die Vertreter^innen der dänischen autonomen Szene) Dren Kastrati eine religiöse Identität, die er gar nicht hat, die noch nicht einmal sein Vater (der vermeintlich ein guter Jugoslawe war) hatte (Arsenijević 2009, 48-49). 
In Arsenijevićs Raubtier-Welt ist für echte Tragik freilich kein Platz, und so schließt auch die Erzählung „Wurzellosigkeit“ nicht mit diesem Schock und dem wenig später folgenden Tod des Helden. Dem Tod wird vielmehr eine ironische Note beigegeben. Dren irrt durch Berlin, er gerät zwischen die Fronten von Autonomen und Polizei und wird im Durcheinander des Gefechts von einem Polizeiwagen überfahren. Marija Pavlović, die ihrerseits vom Zentrum für interkulturellen Dialog deprimiert und betrunken nach Hause zurückkehrt, ist zufällig zur Stelle und nimmt sich des Opfers an. Einer Pietà nicht unähnlich hält sie den sterbenden Dren im Arm und wird in dieser mitleidvollen Haltung von Fotograf*innen erspäht. Die Fotos machen Furore, und so erhält die Geschichte ganz unerwartet ein ironisch markiertes Happy End. Der Tod, das Opfer, der Krieg haben sich zumindest für die Medien - gelohnt.

Arsenijevićs Predator wartet mit mehreren Held^innen auf, die zweifellos Mitleid verdienen, und wir, die Leser`innen, werden im Laufe unserer Lektüre immer wieder auf ihre Seite gezogen. Doch bleiben uns die größeren und kleineren Fehltritte dieser Opfer, ihre egoistischen Kalkulationen und abgründigen Leidenschaften nicht verborgen. Die Opfer, und mit ihnen die Opfer-Verkäufer^innen, unterliegen in Predator ebenso deutlich der Kritik. So bringt Arsenijević, um noch einmal die Terminologie von Käte Hamburger aufzugreifen, ein „ethisch neutrales“ Buch heraus. Durch vielfältige Illusions-, Stil- und Perspektivbrüche, durch provokativ inszenierte, mit Ironie versetzte Gewaltszenen, regt der Autor vor allem das Denken seiner Leserschaft an. Mit dieser intellektuellen Note weist Arsenijević weit über seinen Text hinaus und - im besten Falle - auf die wirklichen Opfer in alten und neuen Zeiten hin. Eine Einschränkung sei jedoch hinzugefügt: Predator zwingt uns wohl kaum dazu, Verantwortung im realen Leben zu übernehmen, der Lektüre Taten folgen zu lassen. Eine solche - ethische und politische - Rezeption wäre zwar möglich, sie drängt sich aber nicht auf. Mit dieser Problematik dürften auch die theoretischen Konzepte, die sich einer Kritik des Mitleids widmen, behaftet sein.

Arsenijevićs unbequeme Schreibweise - die Leser*innen können sich im Mitleid nicht einrichten, alternative Lösungen sind gleichwohl nicht gegeben könnte auch ein Grund für die spärliche Rezeption des Romans sein. Während Arsenijević 1994 mit U potpalublju, einer postmodern-ironischen Antwort auf die beginnenden Kriege in Jugoslawien und die Kriegstreiberei in Serbien, ein großer Wurf gelang - sein Werk wurde in zahlreiche Sprachen, darunter ins Deutsche, Englische und Französische übersetzt ${ }^{31}$-, sieht es mit Predator ganz anders aus.

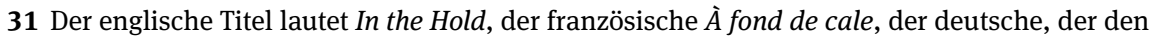
Tetralogie-Titel aufgreift, Cloaca maxima: Eine Seifenoper. 
Das Buch hat es - und das ist ein Erfolg - zumindest ins Albanische geschafft. ${ }^{32}$ Damit hat die internationale Verbreitung aber ihr Bewenden. Im Serbischen gibt es nun eine zweite und sogar eine dritte Auflage. Die Jahre, die seit dem erstmaligen Erscheinen von Predator verstrichen sind, dürften die Aktualität dieser Erzählsammlung durch ihre zahlreichen, politischen ,Flüchtlingskrisen“ unterstrichen haben. Predator hätte eine Übersetzung ins Deutsche ohne Zweifel verdient.

\section{Literaturverzeichnis}

Aristoteles. Poetik. Griech./Dt. Übers. von Manfred Fuhrmann. Stuttgart: Philipp Reclam jun., 1982.

Aristoteles. Poetik. Übers. von Arbogast Schmitt. Berlin: Akademie, 2011.

Arsenijević, Vladimir. „Wurzellosigkeit“. Übers. von Telend [sic] Petrović. Der andere nebenan: Eine Anthologie aus dem Südosten Europas. Hg. Richard Swartz. Frankfurt a. M.:

S. Fischer, 2007. 25-46.

Arsenijević, Vladimir. Predator. Beograd: Samizdat B92, 2009 [2008].

Arsenijeviq, Vladimir. Predatori. Prishtinë: Koha, 2010.

Barthes, Roland. S/Z. Übers. von Jürgen Hoch. Frankfurt a. M.: Suhrkamp, 1976.

Breithaupt, Fritz. „Empathic Sadism: How Readers Get Implicated“. The Oxford Handbook of Cognitive Literary Studies. Hg. Lisa Zunshine. Oxford: Oxford University Press, 2015. 440-462.

Breithaupt, Fritz. Die dunklen Seiten der Empathie. Berlin: Suhrkamp, 2017.

„Center Avnstrup“. Asylzentrum Avnstrup: Gemeinnützige Einrichtung, Kirke Hvalsø, Dänemark, https://www.rodekors.dk/det-goer-vi/roede-kors-asyl/asylcentre/center-avnstrup (25. September 2018).

Fischer, Karsten. „Between Sacrification and Victimization: On Political Semantics and Its Strategic Functions“. Large-Scale Victimisation As a Potential Source of Terrorist Activities. Hg. Uwe Ewald und Ksenija Turković. Amsterdam und Washington, DC: IOS Press, 2006. 67-72.

Foucault, Michel. Überwachen und Strafen: Die Geburt des Gefängnisses. Übers. von Walter Seitter. Frankfurt a. M.: Suhrkamp, 1977.

Fuhrmann, Manfred. Die Dichtungstheorie der Antike: Aristoteles, Horaz, ,Longin‘. Darmstadt: Wissenschaftliche Buchgesellschaft, 1992.

Giglioli, Daniele. Die Opferfalle: Wie die Vergangenheit die Zukunft fesselt. Übers. von Max Henninger. Berlin: Matthes \& Seitz, 2016.

Hamburger, Käte. Das Mitleid. 2. Auflage. Stuttgart: Klett-Cotta, 1996 [1985].

„Kastrioti (Adelsgeschlecht)“. Wikipedia: Die freie Enzyklopädie, https://de.wikipedia.org/wiki/ Kastrioti_(Adelsgeschlecht) (25. September 2018).

Keen, Suzanne. Empathy and the Novel. Oxford: Oxford University Press, 2007.

32 Arsenijeviq 2010. 
Krueger, Joachim. Ästhetik der Antike. Berlin und Weimar: Aufbau, 1983.

Nietzsche, Friedrich. Also sprach Zarathustra: Ein Buch für Alle und Keinen. Berlin und New York: De Gruyter, 1968.

Schadewaldt, Wolfgang. „Furcht und Mitleid? Zu Lessings Deutung des aristotelischen Tragödiensatzes“. Deutsche Vierteljahrsschrift für Literaturwissenschaft und Geistesgeschichte 30 (1956): 137-140.

Schulze Wessel, Martin. „Einleitung“. Opfernarrative: Konkurrenzen und Deutungskämpfe in Deutschland und im östlichen Europa nach dem Zweiten Weltkrieg. Hg. K. Erik Franzen und Martin Schulze Wessel. München: Oldenbourg, 2012.1-8.

Šklovskij, Viktor. O teorii prozy. Moskva: Izd. Federacija, 1929.

Swartz, Richard. Hg. Der andere nebenan: Eine Anthologie aus dem Südosten Europas. Frankfurt a. M.: S. Fischer 2007.

„Vladimir Arsenijević“. Laguna, https://www.laguna.rs/a979_autor_vladimir_arsenijevic_ laguna.html (27. September 2018).

„Vladimir Arsenijević ,Predator““. b92, https://www.b92.net/kultura/moj_ugao.php?nav_ catergory $=559 \& y y y=2009 \& m m=05 \&$ nav_id=324067 (27. September 2018).

Vöhler, Martin. „Die Ambivalenz des Mitleids: Käte Hamburgers Lessing-Kritik“. Ethik und Ästhetik des Mitleids. Hg. Nina Gülcher und Irmela von der Lühe. Freiburg i. Br. u.a.: Rombach, 2007. 33-45.

Zunshine, Lisa. Getting Inside Your Head: What Cognitive Science Can Tell Us about Popular Culture. Baltimore: The Johns Hopkins University Press, 2012.

\section{Filmverzeichnis}

Predator. 20th Century Fox 1987. Regie: John McTiernan. Länge: 107 Min. 
